\title{
Optimal Harvesting in an Integro-difference Population Model
}

\author{
Hem Raj Joshi* Suzanne Lenhart ${ }^{\dagger} \quad$ Holly Gaff ${ }^{\ddagger}$
}

\begin{abstract}
We consider the harvest of a certain proportion of a population that is modeled by an integro-difference equation. This model is discrete in time and continuous in the space variable. The dispersal of the population is modeled by an integral of the population density against a kernel function. The control is the harvest, and the goal is to maximize the profit. The optimal control is characterized by introducing an adjoint function. Numerical results and interpretations are given for four different kernels.
\end{abstract}

Key Words: Hybrid Problem, Optimal Control, Integro-difference Problem

AMS Classification: $\quad 35 \mathrm{~K} 55, \quad 49 \mathrm{~K} 20, \quad 92 \mathrm{D} 25$

\footnotetext{
${ }^{*}$ Xavier University, Mathematics and C S Dept, 3800 Victory Parkway, Cincinnati, OH 45207-4441

${ }^{\dagger}$ Department of Mathematics, University of Tennessee Knoxville, TN 37996-1300

${ }^{\ddagger}$ Dynamics Technology, Inc, 1555 Wilson Blvd, Suite 703, Arlington, VA 22209-2405
} 


\section{Introduction}

Integro-difference equations are models that are discrete in time and continuous in space and possess many of the "spread and growth" attributes of continuous-time reactiondiffusion partial differential equations [4]. These equations model populations with discrete non-overlapping generations and separate growth and dispersal stages [8]. For certain populations, these equations do a better job of estimating the speed of invasion than reaction-diffusion equations [5] and can include a variety of dispersal mechanisms.

We consider the harvest of a certain proportion of the population modeled by the following integro-difference model:

$$
N_{t+1}(x)=\left(1-\alpha_{t}(x)\right) \int_{\Omega} k(x, y) f\left(N_{t}(y), y\right) d y
$$

where $t=0,1, \ldots, T-1$. The state variable $N$ and the control $\alpha$ :

$$
\begin{aligned}
& N=N(\alpha)=\left(N_{0}(x), N_{1}(x), \ldots, N_{T}(x)\right) \\
& \alpha=\left(\alpha_{0}(x), \alpha_{1}(x), \ldots, \alpha_{T-1}(x)\right)
\end{aligned}
$$

represent the population and proportion of the population to be harvested. We use $T$ time steps and $x$ as the spatial variable in a bounded domain $\Omega \subset \mathbb{R}^{n}$. The initial distribution $N_{0}(x)$ is given. We assume $0 \leq \alpha_{t}(x) \leq M<1$ for all $t=0,1, \ldots, T-1$, and $x \in \Omega$. On each time interval, growth occurs first, then dispersal, and finally harvesting. The growth function is represented by $f\left(N_{t}(y), y\right)$. The dispersal of the population is represented by a non-local operator, integration against the kernel $k(x, y)$.

Our goal is to maximize the profit (revenue less cost). The objective functional is defined as:

$$
J(\alpha)=\sum_{t=0}^{T-1} \int_{\Omega}\left[A_{t} e^{-\delta t} \alpha_{t}(x) \int_{\Omega} k(x, y) f\left(N_{t}(y), y\right) d y-\frac{B_{t}}{2}\left(\alpha_{t}(x)\right)^{2}\right] d x .
$$


The coefficient $A_{t}$ gives the price factor and $e^{-\delta t}$ is a discount factor with $\delta>0$. We assume the cost of harvesting is a non-linear function, and here we choose a simple quadratic cost. The coefficient, $B_{t}$, is a weight factor that balances the two parts of the objective functional. Our goal is to find an $\alpha^{*}$ such that

$$
J\left(\alpha^{*}\right)=\max _{\alpha} J(\alpha)
$$

This paper presents an innovative application of optimal control techniques to such an integro-difference model. Optimal control of hybrid systems is a newly developing area and this paper is the first optimal control result in integro-difference equations. This result combines ideas from the discrete version of Pontryagin's Maximum Principle $[3,2,13]$ and optimal control of infinite dimensional systems $[10,11]$ are combined. See also [9] for some hybrid examples. We begin by making the simplifying assumption of linear growth

$$
f\left(N_{t}(y), y\right)=r N_{t}(y)
$$

We realize additional features could be included in the model (like price depending on the population size), but we treat a simple case here to emphasize the novelty in the control techniques. We will treat more general growth functions and other features in subsequent work. See $[7,6,12,5]$ for more background and stability results for integro-difference population models.

In section 2, we state our assumptions and prove the existence of an optimal harvesting control. Section 3 gives the characterization of an optimal control in terms of an optimality system, which is the state equation coupled with an adjoint equation. Uniqueness of an optimal control is given in section 4 under the assumption of the weight factors, $B_{t}$, being sufficiently large. The last section gives numerical results and interpretations for different kernels and discount factors. 


\section{Existence of an Optimal Control}

We assume that

$$
\left|\int_{\mathbb{R}} k(x, y) d x\right| \leq 1
$$

for all $x \in \Omega$ and $0 \leq k(x, y) \leq k_{1}$ for $(x, y) \in \Omega \times \Omega$.

Assuming $M<1$, we define the control set as:

$$
U=\left\{\alpha \in\left(L^{\infty}(\Omega)\right)^{T} \mid 0 \leq \alpha_{t}(x) \leq M, t=0,1, \ldots, T-1\right\}
$$

Assuming $N_{0}(x) \in L^{\infty}(\Omega)$, with $N_{0}(x) \geq 0$, the corresponding state $N=N(\alpha)$ satisfies

$$
0 \leq N_{t}(x) \leq C_{T}
$$

where $C_{T}$ is a constant that depends only on the number of discrete time steps considered in the process. Denote by $\left\|N_{0}\right\|$ the $L^{\infty}$ norm of $N_{0}(x)$, then we obtain

$$
\begin{gathered}
\left\|N_{1}\right\| \leq\left\|\int k r N_{0}\right\| \leq r\left\|N_{0}\right\| \\
\left\|N_{2}\right\| \leq\left\|\int k r N_{1}\right\| \leq r\left\|N_{1}\right\| \leq r^{2}\left\|N_{0}\right\| \\
\left|N_{t+1}(x)\right| \leq\left|1-\alpha_{t}(x)\right| \int_{\Omega}\left|k(x, y) \| r N_{t}(y)\right| d y \\
\leq r^{T}\left\|N_{0}\right\| \int_{\Omega} k(x, y) d y .
\end{gathered}
$$

Theorem 2.1. There exists an optimal control $\alpha^{*}$ in $U$ that maximizes the functional $J(\alpha)$.

Proof: Let $\left\{\alpha^{n}\right\}$ be a maximizing sequence for the objective functional $J$ in (1.2) and $\left\{N^{n}\right\}$ be the corresponding state sequence. Thus on a subsequence

$$
N_{t}^{n} \rightarrow N_{t}^{*} \quad \text { weakly in } L^{2}(\Omega), \quad t=1, \ldots, T
$$




$$
\alpha_{t}^{n}(x) \rightarrow \alpha_{t}^{*} \quad \text { weakly in } L^{2}(\Omega) \quad t=0, \ldots, T-1
$$

since both sequences are a priori $L^{\infty}$ bounded.

We need to show $N^{*}$ is the state corresponding to $\alpha^{*}$, i.e., $N^{*}=N\left(\alpha^{*}\right)$, and the same $\alpha^{*}$ is an optimal control. In this section, we use $\phi(x) \in L^{2}(\Omega)$ as a test function. We multiply both sides of the equation for $N_{t}^{n}$ by the test function and integrate both the sides to obtain:

$$
\int_{\Omega} N_{t+1}^{n}(x) \phi(x) d x=\int_{\Omega}\left(1-\alpha_{t}^{n}\right)(x) \phi(x) \int_{\Omega} k(x, y) r N_{t}^{n}(y) d y d x
$$

Note that

$$
\int_{\Omega} k(x, y) r N_{t}^{n}(y) d y \rightarrow \int_{\Omega} k(x, y) r N_{t}^{*}(y) d y
$$

pointwise for each $x$ in $\Omega$. Boundedness in $L^{\infty}$ of $k(x, y)$ and $N_{t}^{n}(x)$ gives

$$
\phi(x) \int_{\Omega} k(x, y) r N_{t}^{n}(y) d y \rightarrow \phi(x) \int_{\Omega} k(x, y) r N_{t}^{*}(y) d y
$$

strongly in $L^{2}(Q)$. Thus we have

$$
\begin{aligned}
& \int_{\Omega}\left(1-\alpha_{t}^{n}\right)(x) \phi(x) \int_{\Omega} k(x, y) r N_{t}^{n}(y) d y d x \\
& \rightarrow \int_{\Omega}\left(1-\alpha_{t}^{*}\right)(x) \phi(x) \int_{\Omega} k(x, y) r N_{t}^{*}(y) d y d x
\end{aligned}
$$

for each $t=0,1, \ldots, T-1$, since $\left(1-\alpha_{t}^{n}\right)$ is $L^{\infty}$ bounded. Conclude $N^{*}=N\left(\alpha^{*}\right)$.

By the weak $L^{2}$ convergence of $\left\{\alpha_{t}^{n}\right\}$, for each $t=0,1, \ldots, T-1$, we have

$$
\int_{\Omega}\left(\alpha_{t}^{*}(x)\right)^{2} d x \leq \liminf _{n \rightarrow \infty} \int_{\Omega}\left(\alpha_{t}^{n}(x)\right)^{2} d x
$$

This inequality together with the convergence of the integral term discussed above gives

$$
J\left(\alpha^{*}\right) \geq \limsup _{n \rightarrow \infty} J\left(\alpha^{n}\right)
$$

The maximum of $J$ is attained at $\alpha^{*}$. 


\section{Characterization of an Optimal Control}

To characterize an optimal control, we must differentiate the map

$$
\alpha \rightarrow J(\alpha)
$$

which requires first the differentiation of the solution map $\alpha \longrightarrow N=N(\alpha)$. The directional derivative $\psi$ of this solution map is called the sensitivity of the state with respect to the control.

Theorem 3.1. The mapping $\alpha \in U \rightarrow N \in\left(L^{\infty}(\Omega)\right)^{T}$ is differentiable in the following sense:

$$
\frac{N_{t}^{\epsilon}(x)-N_{t}(x)}{\epsilon} \rightarrow \psi_{t}(x)
$$

weakly in $L^{2}(\Omega)$ as $\epsilon \rightarrow 0$ for any $\alpha \in U$ and $l \in L^{\infty}(\Omega)$ such that $(\alpha+\epsilon l) \in U$ for $\epsilon$ small, where $N^{\epsilon}=N(\alpha+\epsilon l)$. Also $\psi$ satisfies:

$$
\begin{aligned}
\psi_{t+1}(x) & =\left(1-\alpha_{t}(x)\right) \int_{\Omega} k(x, y) r \psi_{t}(y) d y-l_{t}(x) \int_{\Omega} k(x, y) r N_{t}(y) d y \\
\psi_{0}(x) & =0 .
\end{aligned}
$$

for $t=0,1, \ldots, T-1$.

Proof: Consider the control-to-solution maps:

$$
\begin{aligned}
& \alpha+\epsilon l \longrightarrow N^{\epsilon}=N(\alpha+\epsilon l) \\
& \alpha \quad \longrightarrow N=N(\alpha) .
\end{aligned}
$$

Since

$$
N_{t+1}^{\epsilon}(x)=\left(1-\alpha_{t}(x)-\epsilon l_{t}(x)\right) \int_{\Omega} k(x, y) r N_{t}^{\epsilon}(y) d y,
$$


we have

$$
\begin{aligned}
\frac{N_{t+1}^{\epsilon}(x)-N_{t+1}(x)}{\epsilon}= & \left(1-\alpha_{t}(x)\right) \int_{\Omega} k(x, y)\left[\frac{r N_{t}^{\epsilon}(y)-r N_{t}(y)}{\epsilon}\right] d y \\
& -l_{t}(x) \int_{\Omega} k(x, y) r N_{t}^{\epsilon}(y) d y
\end{aligned}
$$

Using $N_{0}^{\epsilon}=N_{0}$ and

$$
\frac{N_{1}^{\epsilon}(x)-N_{1}(x)}{\epsilon}=-l_{0} \int_{\Omega} k(x, y) r N_{0}(y) d y
$$

we obtain,

$$
\left|\frac{N_{1}^{\epsilon}(x)-N_{1}(x)}{\epsilon}\right| \leq C_{1} \quad \text { for all } x \in \Omega
$$

and then by iteration,

$$
\left|\frac{N_{t}^{\epsilon}(x)-N_{t}(x)}{\epsilon}\right| \leq C_{2} \quad \text { for all } x \in \Omega, t=1,2, \ldots T .
$$

For all $t=1,2, \ldots T$, on a subsequence, we have the convergence

$$
\frac{N_{t}^{\epsilon}(x)-N_{t}(x)}{\epsilon} \rightarrow \psi_{t} \quad \text { weakly in } L^{2}(\Omega)
$$

Similar to the proof of Theorem 2.1, we obtain $\psi$ satisfies (3.1).

Theorem 3.2. Given an optimal control $\alpha^{*}$ and corresponding state solution $N^{*}=$ $N\left(\alpha^{*}\right)$, there exists a weak solution $p \in\left(L^{\infty}(\Omega)\right)^{T}$ satisfying the adjoint system:

$$
\begin{aligned}
p_{t-1}(x) & =r \int_{\Omega}\left(1-\alpha_{t-1}^{*}(y)\right) k(y, x) p_{t}(y) d y+r \int_{\Omega} A_{t-1} e^{-\delta(t-1)} \alpha_{t-1}^{*}(y) k(y, x) d y \\
p_{T}(x) & =0
\end{aligned}
$$

where $t=T, \ldots 2,1$. Furthermore, for $t=0,1,2, \ldots, T-1$

$$
\alpha_{t}^{*}=\min \left(\max \left(\left(\frac{1}{B_{t}}\left(A_{t} e^{-\delta t}-p_{t+1}(x)\right) \int_{\Omega} k(x, y) r N_{t}^{*}(y) d y\right), 0\right), M\right) .
$$


Proof: Let $\alpha^{*}$ be an optimal control (which exists by Theorem 2.1) and $N(\alpha)$ be its corresponding state. Let $\left(\alpha^{*}+\epsilon l\right) \in U$ for $\epsilon>0$, and $N^{\epsilon}$ be the corresponding solution of the state equation (1.1). Since the adjoint system is linear, there exists a weak solution $p$ satisfying (3.2). We compute the directional derivative of the functional $J(\alpha)$ with respect to $\alpha$ in the direction $l$. Since $J\left(\alpha^{*}\right)$ is the maximum value, we have

$$
\begin{aligned}
0 \geq & \lim _{\epsilon \rightarrow 0^{+}} \frac{J\left(\alpha^{*}+\epsilon l\right)-J\left(\alpha^{*}\right)}{\epsilon} \\
=\lim _{\epsilon \rightarrow 0^{+}} & \sum_{t=0}^{T-1} \frac{1}{\epsilon}\left[\int_{\Omega}\left(A_{t} e^{-\delta t}\left(\alpha^{*}+\epsilon l\right)_{t}(x) \int_{\Omega} k(x, y) r N_{t}^{\epsilon}(y) d y-\frac{B_{t}}{2}\left(\left(\alpha^{*}+\epsilon l\right)_{t}\right)^{2}(x)\right) d x\right. \\
& \left.\quad-\int_{\Omega}\left(A_{t} e^{-\delta t} \alpha_{t}^{*}(x) \int_{\Omega} k(x, y) r N_{t}^{*}(y) d y-\frac{B_{t}}{2}\left(\alpha_{t}^{*}(x)\right)^{2}\right) d x\right] \\
= & \lim _{\epsilon \rightarrow 0^{+}} \sum_{t=0}^{T-1}\left\{\int_{\Omega} A_{t} e^{-\delta t} \alpha_{t}^{*}(x) \int_{\Omega} k(x, y)\left[\frac{r N_{t}^{\epsilon}(y)-r N_{t}^{*}(y)}{\epsilon}\right] d y d x\right. \\
& \left.+\int_{\Omega}\left[A_{t} e^{-\delta t} l_{t}(x) \int_{\Omega} k(x, y) r N_{t}^{\epsilon}(y) d y-B_{t} \alpha_{t}^{*} l_{t}\right] d x\right\} \\
= & \sum_{t=0}^{T-1}\left\{\int_{\Omega} A_{t} e^{-\delta t} \alpha_{t}^{*}(x) \int_{\Omega} k(x, y) r \psi_{t}(y) d y d x\right. \\
& \left.+\int_{\Omega} A_{t} e^{-\delta t} l_{t}(x) \int_{\Omega} k(x, y) r N_{t}^{*}(y) d y d x-\int_{\Omega} B_{t} \alpha_{t}^{*} l_{t} d x\right\} \\
= & \sum_{t=0}^{T-1}\left\{\int_{\Omega} r \psi_{t}(y) \int_{\Omega} A_{t} e^{-\delta t} \alpha_{t}^{*}(x) k(x, y) d x d y\right. \\
& \left.+\int_{\Omega} A_{t} e^{-\delta t} l_{t}(x) \int_{\Omega} k(x, y) r N_{t}^{*}(y) d y d x-\int_{\Omega} B_{t} \alpha_{t}^{*} l_{t} d x\right\}
\end{aligned}
$$

where the last equality is obtained by switching the order of integration on first integral. We use the coefficient of the $\psi_{t}$ term as the non-homogeneous term in the adjoint system. Substituting from the adjoint system (3.2), we simplify the first term in the $J$ deriva- 
tive expression

$$
\begin{aligned}
& \sum_{t=0}^{T-1} \int_{\Omega} r \psi_{t}(y) \int_{\Omega} A_{t} e^{-\delta t} \alpha_{t}^{*}(x) k(x, y) d x d y \\
& =\sum_{t=0}^{T-1}\left\{\int_{\Omega} \psi_{t}(y)\left[p_{t}(y)-r \int_{\Omega}\left(1-\alpha_{t}^{*}(x)\right) k(x, y) p_{t+1}(x) d x d y\right]\right\} \\
& =\sum_{t=0}^{T-1} \int_{\Omega} p_{t+1} \psi_{t+1}(x) d x-\sum_{t=0}^{T-1} \int_{\Omega} p_{t+1}(x)\left(1-\alpha_{t}^{*}(x)\right) \int_{\Omega} k(x, y) r \psi_{t}(y) d y d x
\end{aligned}
$$

In the last equality, we rewrote first sum, added $p_{T} \psi_{T}$ term, and used $p_{T}=0, \psi_{0}=0$.

Then using the $\psi$ system, (3.4) becomes (3.1):

$$
\begin{aligned}
& \sum_{t=0}^{T-1} \int_{\Omega} p_{t+1}(x)\left[\psi_{t+1}(x)-\left(1-\alpha_{t}(x)\right) \int_{\Omega} k(x, s) r \psi_{t}(s) d s\right] d x \\
& =\sum_{t=0}^{T-1} \int_{\Omega} p_{t+1}(x)\left(-l_{t}(x) \int_{\Omega} k(x, y) r N_{t}^{*}(y) d y\right) d x .
\end{aligned}
$$

We go back to differentiating $J$, substituting out for first term from the above $p, \psi$ calculation,

$$
\begin{aligned}
& 0 \geq \sum_{t=0}^{T-1}\left\{\int_{\Omega} r \psi_{t}(y) \int_{\Omega} A_{t} e^{-\delta t} \alpha_{t}^{*}(x) k(x, y) d x d y\right. \\
& \left.\quad+\int_{\Omega} A_{t} e^{-\delta t} l_{t}(x) \int_{\Omega} k(x, y) r N_{t}^{*}(y) d y d x-\int_{\Omega} B_{t} \alpha_{t}^{*} l_{t} d x\right\} \\
& =\sum_{t=0}^{T-1} \int_{\Omega} l_{t}(x)\left[-p_{t+1}(x) \int_{\Omega} k(x, y) r N_{t}^{*}(y) d y-B_{t} \alpha_{t}^{*}(x)\right. \\
& \left.\quad+A_{t} e^{-\delta t} \int_{\Omega} k(x, y) r N_{t}^{*}(y) d y\right] d x .
\end{aligned}
$$

For $t=0,1, \ldots, T-1$, we obtain:

$$
\alpha_{t}^{*}=\frac{1}{B_{t}}\left(A_{t} e^{-\delta t}-p_{t+1}(x)\right) \int_{\Omega} r k(x, y) N_{t}^{*}(y) d y
$$


subject to the control bounds. Choosing appropriate choices of variation $l$ on the size of $\alpha^{*}$ we obtain the representation $(3.3)$

The optimal control satisfies an integro-difference condition involving optimal state and adjoint variables.

The optimality system is (1.1) and (3.2) with $N_{0}(x)$ known, $p_{T}(x)=0$, and the characterization condition (3.3).

\section{Uniqueness Result}

Theorem 4.1. If $B_{t}, t=0,1, \ldots, T-1$ are sufficiently large, then the optimal control is unique.

Proof. We show uniqueness by showing strict concavity of the map:

$$
\alpha \in U \longrightarrow J(\alpha)
$$

The concavity follows from showing for all $\alpha, l \in U, 0<\epsilon<1$

$$
g^{\prime \prime}(\epsilon)>0
$$

where $g(\epsilon)=J(\epsilon l+(1-\epsilon) \alpha)=J(\alpha+\epsilon(l-\alpha))$.

First, we calculate

$$
\begin{aligned}
g^{\prime}(\epsilon)= & \lim _{\tau \rightarrow 0} \frac{J(\alpha+(\epsilon+\tau)(l-\alpha))-J(\alpha+\epsilon(l-\alpha))}{\tau} \\
= & \sum_{t=0}^{T-1}\left[\int_{\Omega} A_{t} e^{-\delta t}\left(\alpha_{t}+\epsilon\left(l_{t}-\alpha_{t}\right)\right) \int_{\Omega} r k(x, y) \psi_{t}^{\epsilon}(y) d y d x\right. \\
& +\int_{\Omega} A_{t} e^{-\delta t}\left(l_{t}-\alpha_{t}\right) \int_{\Omega} r k(x, y) N_{t}^{\epsilon}(y) d y d x \\
& \left.-B_{t} \int_{\Omega}\left(\alpha_{t}+\epsilon\left(l_{t}-\alpha_{t}\right)\right)\left(l_{t}-\alpha_{t}\right) d x\right]
\end{aligned}
$$


where, for $t=0,1, \ldots, T-1$

$$
\begin{aligned}
& N^{\epsilon}=N(\alpha+\epsilon(l-\alpha)) \\
& N^{\epsilon, \tau}=N(\alpha+(\epsilon+\tau)(l-\alpha)) \\
& \frac{N_{t}^{\epsilon, \tau}-N_{t}^{\epsilon}}{\tau} \rightarrow \psi_{t}^{\epsilon} \quad \text { as } \tau \rightarrow 0 \\
& \psi_{t+1}^{\epsilon}(x)=\left(1-\left(\alpha_{t}+\epsilon\left(l_{t}-\alpha_{t}\right)\right)\right)(x) \int_{\Omega} r k(x, y) \psi_{t}^{\epsilon} d y \\
& \quad-\left(l_{t}-\alpha_{t}\right) \int_{\Omega} r k(x, y) N_{t}^{\epsilon} d y \\
& \psi_{0}^{\epsilon} \equiv 0 .
\end{aligned}
$$

Note that $\psi_{t}^{\epsilon}(x)$ can be estimated in terms of $l-\alpha$. For example,

$$
\left|\psi_{2}^{\epsilon}(x)\right| \leq C_{1}\left[\int_{\Omega}\left|l_{0}-\alpha_{0}\right| d y+\left|l_{1}-\alpha_{1}\right|(x)\right],
$$

where $C_{1}$ depends on $L^{\infty}$ bounds on the states, $k(x, y)$ and $r$. One can continue to estimate iteratively.

Next we obtain

$$
\begin{aligned}
g^{\prime \prime}(\epsilon)= & \sum_{t=0}^{T-1}\left[\int_{\Omega} A_{t} e^{-\delta t}\left(\alpha_{t}+\epsilon\left(l_{t}-\alpha_{t}\right)\right) \int_{\Omega} r k(x, y) \sigma_{t}^{\epsilon}(y) d y d x\right. \\
& \left.+2 \int_{\Omega} A_{t} e^{-\delta t} \epsilon\left(l_{t}-\alpha_{t}\right) \int_{\Omega} r k(x, y) \psi_{t}^{\epsilon}(y) d y d x-B_{t} \int_{\Omega}\left(l_{t}-\alpha_{t}\right)^{2} d x\right]
\end{aligned}
$$

where, for $t=0,1, \ldots, T-1$

$$
\begin{aligned}
& \sigma_{t+1}^{\epsilon}(x)=\left(1-\left(\alpha_{t}+\epsilon\left(l_{t}-\alpha_{t}\right)\right)\right)(x) \int_{\Omega} r k(x, y) \sigma_{t}^{\epsilon} d y-2\left(l_{t}-\alpha_{t}\right) \int_{\Omega} r k(x, y) \psi_{t}^{\epsilon} d y \\
& \sigma_{0}^{\epsilon} \equiv 0 .
\end{aligned}
$$


We can estimate

$$
\int_{\Omega}\left|\sigma_{t}^{\epsilon}(y)\right| d y
$$

in terms of

$$
\int_{\Omega}\left(l_{k}-\alpha_{k}\right)^{2} d y, \quad k=0,1, \ldots, t-1 .
$$

Using the estimates,

$$
g^{\prime \prime}(\epsilon) \leq \sum_{t=0}^{T-1}\left(C_{2}-\epsilon B_{t}\right) \int_{\Omega}\left(l_{t}-\alpha_{t}\right)^{2} d y,
$$

which gives the desired concavity for $B_{t}$ 's sufficiently large.

\section{$5 \quad$ Numerical Results}

Dispersion kernels have been measured for a wide variety of organisms. The resulting data display an incredible variety of patterns and strategies. Some dispersal kernels have been found to be leptokurtic (fat-tailed) rather than normal [6]. We explored the following four dispersal kernels. A finite range dispersal kernel [7]

$$
k(x, y)= \begin{cases}0, & x \leq y-R, \\ \frac{\pi}{4 R} \cos \left[\frac{\pi}{2 R}|x-y|\right], & y-R \leq x \leq y+R, \\ 0, & x \geq y+R .\end{cases}
$$

may be appropriate if the surrounding environment is incompatible with the organism being studied. The Laplace kernel [4],

$$
k(x, y)=\frac{1}{2} \beta \exp (-\beta|x-y|),
$$


Figure 1: Graph of all Kernel

or normal dispersal [6],

$$
k(x, y)=\sqrt{\frac{\beta}{\pi}} \exp \left(-\beta(x-y)^{2}\right),
$$

are reasonable choices for certain populations. The biology of some organisms results in a radically different dispersal pattern. The ballistic dispersal,

$$
k(x, y)=\frac{3 a}{2}|x-y|^{b-1} \exp -a|x-y|^{3}
$$

is appropriate for propagules that are launched by the parent organism [12]. Figure 1 shows the dispersal pattern for each of the four examples for an organism located at 0.5.

One of the most challenging aspects of calculating the solution for this system of equations is estimating the spatial grid needed for accuracy. We use the composite trapezoid rule to estimate each integral [1]. The number of points needs to be large enough to minimize error but not so large that the run time is bogged down with needless calculations. The dispersal kernel chosen has the most influence over the spatial mesh size needed for accuracy. For each kernel, we estimated the spatial mesh size by solving the forward equations for $N$ alone without control. We repeat this estimation using a progressively finer mesh size until the differences between estimates for $N$ were less than one percent. This mesh size was then used to set up the numerical solution for the optimal control problem.

The search for the optimal control uses an iterative process. With the initial estimates for the adjoint, $p, N_{0}$ and $\alpha$, the iterative process begins by solving the equations for $N$ forward in time. Then using these new estimates for $N$, new $p$ values are calculated backwards through time. The control $\alpha$ is updated using a convex combination of the old value and the value calculated from characterization (3.3). The new estimates of $N$ 
and $p$ are compared with those from the previous iteration. If the difference is less than one percent for every point, the final control $\alpha$ is saved as the optimal solution.

For each of our numerical solutions, we used 10 time steps. We also assumed that $\Omega=[0,1]$, which was divided into 5000 spatial grid points for the trapezoid rule. The figures are generated using following parameter values (unless stated in the captions): $r=1.8, A_{t}=1.0, B_{t}=1600, \delta=0.1, \alpha_{0}=0.1, N_{0}(x)=75.0$, and for kernels $R=0.5, \beta=$ $5.0, a=250, b=3$. A number of different initial conditions including $N_{0}(x)=1-x$ and $N_{0}(x)=x(1-x)$ were tested, but the results were not significantly different. We found the shape of the optimal solution to be quite sensitive to the discount rate; unless otherwise noted we took $\delta=0.35$.

Figure 2 shows the optimal harvesting routine as calculated by the numerical simulation using the kernel given by (5.1). Figure 3 shows the population that results from the optimal harvesting scheme of Figure 2. The Laplace, normal and finite-range kernels were found to be qualitatively similar enough to produce similar optimal solutions. Figures 4 and 5 show variation in the shape of the optimal solution based on the ballistic dispersal kernel. Figures 6 and 7 show that changes in the discount rate radically changes the harvesting strategy. A very high discount rate results in early harvesting (Figure 6) while a very low discount rate results in higher harvesting in the last few time steps (Figure 7). This makes sense since after such early harvest, one would invest the money taking advantage of the high interest rate. It can be be noted that for kernels (5.1 - 5.3), the harvest begins on the edges of the domain earlier than the middle. This is a result of the relatively lower contribution to overall population size from the population on the edges from dispersal. 
Figure 2: Harvest Rate for Kernel 1

Figure 3: Population for Kernel 1

Figure 4: Harvest Rate for Kernel 4

Figure 5: Population for Kernel 4

Figure 6: Harvest Rate for Kernel 1 with 0.35 discount rate

Figure 7: Harvest Rate for Kernel 1 with 0.05 discount rate 
These results are also highly dependent on the growth function used. Using a growth function that includes a carrying capacity could result in more variable optimal solutions. These types of problems will be pursued in future work.

Acknowledgment: This work was partially supported by National Science Foundation grant DMS 0110920.

\section{References}

[1] Burden, R. L. and Faires, J. D., Numerical Analysis, PWS Publishing Company, Boston, 1993.

[2] Fan, L. and Wang, C., The Discrete Maximum Principle, John Wiley, New York, 1964.

[3] Kamien, M. I. and Schwarz, N. L., Dynamic Optimization, North-Holland, Amsterdam 1991.

[4] Kot, M., Discrete-time Traveling Waves: Ecological Examples, J. Math. Biol. (1992) 30: 413-436.

[5] Kot, M., Do Invading Organisms Do the Wave?, Canadian Applied Mathematics Quarterly, 2003. In press.

[6] Kot, M., Lewis, M. A., and Driessche, P. van den, Dispersal Data and the Spread of Invading Organisms, Ecology (1996) 77: 2027-2042.

[7] Kot, M. and Schaffer, W. M., Discrete-Time Growth-Dispersal Models, Mathematical Biosciences (1986) 80: 109-136. 
[8] Lewis, M. A. and Van Kirk, R. W., Integrodifference Models for Persistence in Fragmented Habitats, Bull. Math. Biology (1997) 59: 107-137.

[9] Lenhart S. and Protopopescu V., Modeling and Analysis in Support of Decision Making for Technological Investment, Oak Ridge National Laboratory Technical Memo ORNL/TM-2003/116.

[10] Li, X. and Yong, J., Optimal Control Theory for Infinite Dimensional Systems, Birkhaüser, Boston 1995

[11] Lions, J. L., Optimal Control of Systems Governed by Partial Differential Equations, Springer-Verlag, New York, 1971.

[12] Neubert, M. G., Kot, M. and Lewis, M. A., Dispersal and pattern formation in a discrete-time predator-prey model, Theoretical Population Biology (1995) 48: 7-43.

[13] Sethi, S. and Thompson. G. L., Optimal Control Theory: Applications to Management Science and Economics, Kluwer Academic, Boston, 2000. 